\title{
VALEUR COMPARÉE DES PHOSPHATES MINÉRAUX COMME SOURCES DE PHOSPHORE POUR LES ANIMAUX
}

\author{
I. ('THGUEN \\ Service de Biahimie et de Nutrition, \\ Centre national de Recherches sootechniques, Juny-ent-Josas (Seine et Oisi).
}

\section{SOMMAIRE}

L'utilisation du phosphore d'un phosphate peut varier en fonction de divers facteurs propres it l'animal, au mode d'alimentation et au produit lui-même. Il est donc difficile d'apprécier, même par un choix convenable de critères, la valeur nutritionnelle d'un phosphate.

Cette mise au point rassemble les principaux résultats concernant la valeur comparée des phosphates minéraux comme sources de phosphore pour les animaux. Tes nombreuses contradictions apparentes peuvent souvent s'expliquer par les différences entre les moyens et les méthodes mis en œuvre pour apprécier la valeur du produit.

Toutefois, il existe manifestement entre phosphates des différences qui ne peuvent être attribuées qu'à la nature même du produit : finesse des particules, forme chimique et degré de polymérisation, forme cristalline, traitement subi avant l'emploi et autres constituants du phosphate. Le cas des phosphates contenant une grande quantité de fer et d'aluminium a été particulièrement étudié.

Il est également possible d'évaluer approximativement l'efficacité biologique d'un phosphate par des méthodes in vitro.

Si les orthophosphates purs, sources classicues de phosphore, sont toujours bien utilisés par tous les animaux, en revanche l'efficacité des phosphates naturels bruts ou défluorés est très variable tandis que les phosphates alumino-ferriques sont en général les plus mal utilisés, en particulier par les monogastriques.

Parmi les problèmes que pose l'alimentation minérale des animaux, celui de l'apport du phosphore est en général le plus aigu, non seulement parce que les rations de base sont souvent insuffisamment pourvues en phosphore et que les carences ont des conséquences graves, mais aussi, parce que le coût du complément phosphaté est très variable et en général assez élevé.

Le besoin impérieux de réduire le prix de revient du complément phosphaté a conduit à la multiplicité actuelle des phosphates du commerce. Cette grande variété permet en particulier de choisir des phosphates pauvres en calcium, ce qui peut être utile dans le cas des ruminants recevant des rations à base de légumineuses (excédentaires en calcium).

Il est bien connu que le phosphore d'origine minérale est aussi bien, et même parfois mieux utilisé par les animaux que le phosphore provenant des aliments naturels (ceci est particulièrement vrai pour les monogastriques qui utilisent mal le phosphore phytique des céréales). 
Cependant, la hiérarchie qui existe probablement dans les efficacités biologiques des différentes sources minérales de phosphore est mal connue et, bien que ces comparaisons aient fait l'objet d'innombrables travaux, il existe encore dans ce domaine de nombreuses contradictions, sinon des inconnues.

\section{I. - LES MÉCANISMES DE L'U'IILISATION DU PHOSPHORE PAR L'ORGANISME}

Sans entrer dans le détail des processus du métabolisme du phosphore, il importe de rappeler brièvement ses principales étapes et de définir la terminologie adoptée pour apprécier le degré d'utilisation du phosphore par l'animal.

La digestibilité apparente, ou coefficient d'utilisation digestive apparent, est la différence entre la quantité de $\mathrm{P}$ ingérée et la quantité totale de $\mathrm{P}$ excrétée par les fèces. Cette différence est rapportée à Ioo parties de $\mathrm{P}$ ingéré et mesure l'absorption relative apparente.

$$
\text { C. U. I. apparent }=\frac{P \text { ingéré }-P \text { fécal }}{P} \times \text { Ioo }
$$

Le coefficient de rétention apparent, particulièrement employé chez les animaux en croissance, est la différence (rapportée à Ioo parties de $\mathrm{P}$ ingéré), entre la quantité de $\mathrm{P}$ ingérée et la quantité totale de $\mathrm{P}$ excrétée (fèces + urine).

$$
\text { C. } \mathrm{R} \text {. apparent }=\frac{\mathrm{P} \text { ingéré }-(\mathrm{P} \text { fécal }+\mathrm{P} \text { urinaire })}{\mathrm{P} \text { ingéré }} \times \text { Ioo }
$$

Notons dès à présent que chez les ruminants adultes l'excrétion urinaire de $\mathrm{P}$ est en général négligeable et le coefficient de rétention est donc du même ordre que la digestibilité apparente ; toutefois, chez les femelles laitières, l'excrétion urinaire de $\mathrm{P}$ peut atteindre Io à $\mathrm{I} 5 \mathrm{p}$. Ioo de l'excrétion totale durant les premières semaines de lactation (BROCHART, Ig6I).

Le bilan est la différence entre la quantité de $\mathrm{P}$ ingérée et la quantité totale de $\mathrm{P}$ perdue par l'organisme (fèces, urine, lait). C'est pourquoi cette expression est le plus souvent utilisée dans le cas des femelles laitières, car le phosphore préalablement retenu pour la formation du lait constitue cependant une perte pour l'organisme.

Le phosphore absorbé par la paroi intestinale se répartit en plusieurs fractions : $P$ retenu (fixation osseuse en particulier), $\mathrm{P}$ excrété dans l'urine, éventuellement $\mathrm{P}$ sécrété dans le lait et enfin $\mathbf{P}$ réexcrété dans le tractus digestif et se retrouvant donc dans les fèces aù même titre que le $\mathrm{P}$ non digéré provenant directement de l'aliment. Cette fraction du $\mathrm{P}$ des fèces (provenant en majeure partie des échanges effectués au niveau de l'os), déjà utilisée à une fin métabolique, est appelée $\mathrm{P}$ endogène fécal et ne peut être appréciée avec certitude que par l'emploi des isotopes radioactifs. Connaissant la partie d'origine endogène du $\mathrm{P}$ fécal il est possible de déterminer la digestibilité vraie ou coefficient d'utilisation digestive réel du $\mathrm{P}$ ingéré.

$$
\text { C. U. I. réel }=\frac{\mathrm{P} \text { ingéré }-(\mathrm{P} \text { fécal }-\mathrm{P} \text { endogène })}{\mathrm{P} \text { ingéré }} \times \text { Ioo }
$$


De même, en tenant compte dı phosphore endogène fécai (dont la majeure partie provient des échanges au niveau de l'os) on obtient la rétention réelle du phosphore alimentaire.

Pour interpréter les résultats obtenus à l'aide des méthodes de bilans, il faut admettre 1'hypothèse, d'ailleurs discutable, de la dissociation des métabolismes exogène et endogène du phosphore. Ainsi, on peut analyser les variations de l'utilisation du $\mathrm{P}$ de la ration par l'étude de plusieurs séries de facteurs bien distincts (ne pouvant être dissociés expérimentalement avec certitude que par l'emploi de composés marqués par ${ }^{32} \mathrm{P}$ ) agissant soit sur l'absorption (surtout la solubilité dans les conditions de l'intestin), soit sur la réexcrétion, ou soit encore sur la mobilisation des réserves osseuses.

\section{II. - LES DIFFICULTÉS D'ÉVALUATION DE L'EFFICACITÉ BIOLOGIQUE DES PHOSPHATES}

La mesure des différences entre les efficacités biologiques de plusieurs phosphates est une opération très délicate. Ceci est dû, d'une part à la multiplicité des facteurs susceptibles d'influer sur l'utilisation physiologique du phosphore, et d'autre part au choix des méthodes et critères d'évaluation.

I $^{\circ}$ Les facteurs agissant sur l'utilisation du phosphore par les animaux.

\section{Facteurs propres à l'animal.}

Les besoins de l'animal, déterminés par son état physiologicule (croissance, gestation, lactaction, entretien) et son âge, influent bien entendu sur l'utilisation du $\mathrm{P}$ alimentaire. C'est pourquoi le phosphore d'une même ration est bien mieux retenu par un jeune animal en pleine croissance par exemple que par un animal adulte à l'état d'entretien.

L'état des réserves osseuses, qui traduit le passé nutritionnel de l'animal, influe également sur ses besoins en phosphore. Ainsi, un état de déplétion préalable de l'animal favorise l'utilisation du phosphore de la ration ; 1'état des réserves osseuses agirait surtout, selon HANSARD et al (I957), sur la rétention du phosphore absorbé et non pas sur l'absorption intestinale.

Il existe en outre, comme nous le verrons plus loin, des différences considérables entre espèces, et en particulier entre ruminants et monogastriques, pour ce qui concerne le métabolisme des éléments minéraux. Il n'est donc pas possible de généraliser les résultats obtenus sur une espèce.

Enfin, les difficultés de comparaison des efficacités biologiques du phosphore de plusieurs sources sont encore accrues par la variabilité des capacités individuelles d'assimilation.

\section{Facteurs liés à la composition de la ration.}

Tout d'abord, l'utilisation du phosphore de la ration varie en sens inverse de la quantité totale de phosphore ingérée. L'adaptation à des niveaux d'ingestion variable, bien étudiée dans le cas du calcium, où le rôle homéostatique de l'intestin 
a été démontré par HARRISOr (1959), semble plus complexe dans le cas du phosphore : des variations de l'ingestion entraîneraient simultanément des modifications de l'absorption intestinale, de l'excrétion urinaire et de l'excrétion endogène fécale.

D'autre part, pour apprécier l'utilisation du phosphore apporté par le complément, il est souvent difficile de faire abstraction du phosphore apporté par la ration de base ; c'est pourquoi les valeurs trouvées pour l'efficacité d'un phosphate comme source de $\mathrm{P}$ dépendent de la proportion de $\mathrm{P}$ apportée par la ration de base dans l'expérience.

Les autres constituants de la ration peuvent influer considérablement sur l'utilisation de $\mathrm{P}$. Ainsi la vitamine $\mathrm{D}$ agirait de façon indirecte (action primaire sur l'absorption intestinale du calcium) sur l'absorption de $\mathrm{P}$ et agirait en outre directement surla fixation ossense et l'excrétion urinaire de P (WASSERMAN et al. I960). Quant au déséquilibre phospho-calcique de la ration, il semble que son effet néfaste ait souvent été exagéré. Ainsi, dans le cas des ruminants, le rapport $\mathrm{Ca} / \mathrm{P}$ peut varier entre I et 6 sans que le métabolisme de $P$ soit perturbé (I)owE et al. I957) et sans qu'il y ait apparition de troubles de fertilité (LiTTr.EJOHx et al. I960) à condition que l'apport de $\mathrm{P}$ soit conforme aux besoins. Luliken et a1. (Ig6I) utilisant la méthode de dilution isotopique pour $\mathrm{Ca}$ et $\mathrm{P}$ sur des agneaux en croissance, ont bien montré qu'une variation du rapport $\mathrm{Ca} / \mathrm{P}$ entre 0,8 et 6 n'avait aucune influence propre sur l'absorption de ces deux éléments. Même chez les monogastriques, des travaux récents sur rats (HARRIS I959, CACSERET et al. I958) conduisent à diminuer l'importance attribuée à un rapport $\mathrm{Ca} / \mathrm{P}$ trop élevé et à un excès de $\mathrm{Ca}$ dans la ration, si l'apport de $\mathrm{P}$ est suffisant. En revanche, un rapport $\mathrm{Ca} / \mathrm{P}$ trop faible peut nuire à l'utilisation du phosphore dans la mesure où l'apport de calcium est insuffisant tet ne permet pas la fixation maximum de phosphore (les fixations osseuses de Ca et $\mathrm{P}$ s'effectuent simultanément et dans un rapport voisin de 2). Ceci expliquerait les différences enregistrées sur porcs par Combs et al. (Ig60) pour des rapports $\mathrm{Ca} / \mathrm{P}$ variant de 0,9 à 2 . Il est également bien connu que la présence de vitamine 1 ) est nécessaire pour l'adaptation à un déséquilibre phospho-calcique de la ration.

Enfin, tous les facteurs susceptibles de modifier la vitesse du transit dans le tractus digestif (teneur en eau, teneur en cellulose...) doivent jouer un rôle important dans l'absorption de $P$.

\section{Facteurs propres an phosphate.}

En réalisant des conditions identiques d'utilisation, compte tenu des facteurs de variation énumérés ci-dessus, il existe cependant des différences entre les sources de $\mathrm{P}$. Ces différences, parfois importantes, ne peuvent être attribuées qu'à la nature même du produit, et nous nous proposons de les étudier dans la discussion des résultats.

$2^{\circ}$ Moyens d'évaluation

du coefficient d'utilisation du phosphore des phosphates.

Les divergences qui existent entre les résultats des nombreux travaux concernant l'efficacité biologique des phosphates sont en général dues aux différences entre les méthodes et les critères itilisés. 
Méthodes utilisées pour la comparaison des phosphates.

La plupart des essais de classification des phosphates ont été effectués d'après des expériences in vivo, où l'on a comparé les résultats obtenus sur plusieurs animaux recevant divers compléments. Toutefois, certaines classifications résultent de comparaisons in vitro, par exemple par la technique du rumen artificiel (ANDERson et al. 1956) ou plus simplement par des différences de solubilité dans des solvants appropriés (HILI, et al I945, ELIIS et al. I945, CABEII, et al. I950).

Dans les expériences sur animaux, les résultats obtenus diffèrent en général suivant les techniques utilisées. Ainsi, les animaux présentant de gros besoins, soit à cause de leur état physiologique (lactation, croissance), soit à la suite d'une déplétion préalable, constituent le meilleur matériel pour les comparaisons entre les efficacités biologiques de plusieurs phosphates. D'autre part, le coefficient d'utilisation du P du complément varie suivant la proportion du $\mathrm{P}$ fourni par la ration de base dans le $\mathrm{P}$ total ingéré. Ainsi, les expériences utilisant des régimes semi-purifiés, dont la ration de base est très pauvre en $P$, fournissent des différences plus fortes entre compléments. De plus, lorsque la ration de base fournit une quantité de $\mathrm{P}$ trop proche des besoins minima de l'animal, l'appréciation de la valeur du complément est nécessairement fausse.

Enfin, il est difficile de comparer des expériences utilisant des phosphates de même nature et apparemment identiques; selon leur origine ou leur traitement ils peuvent en effet être très différents : c'est le cas des phosphates défluorés en particulier.

\section{Critives d'appréciation de l'utilisation du phosphore.}

Les principaux critères utilisés dans les travaux sur l'utilisation des phosphates sont les suivants : la vitesse de croissance, l'efficacité alimentaire, la teneur en $\mathrm{P}$ du sérum, les teneurs en cendres et en $\mathbf{P}$ du fémur ou du tibia, le poids du tibia sec dégraissé rapporté au poids du corps, l'examen radiographique et l'étude de la résistance de certains os (fémur ou os de la mâchoire), et enfin, la méthode des bilans. Par cette dernière méthode on peut obtenir, soit setlement la rétention nette et l'absorption apparente, soit également l'excrétion fécale endogène de $\mathrm{P}$ et l'absorption vraie (au moyen de techniques utilisant les isotopes radioactifs).

Comme nous le verrons plus loin, les résultats et leur interprétation varient suivant le groupe de critères choisis. D'autre part, certains critères n'ont qu'une valeur limitée : ainsi le taux du P sérique ne pent être un reflet valable de l'utilisation nette du phosphore, celui-ci pouvant dans certains cas être absorbé, puis être réexcrété et non retenu (AMmERMAN et a1. I957).

\section{III. - RÉSULTATS}

Il est très difficile de rassembler et de comparer des résultats obtenus sur des proluits si variés et par des méthodes souvent différentes. C'est pourquoi nous diviserons d'abord les travaux en quatre séries suivant les espèces ou groupes d'espèces étudiés : les rats, les volailles, les porcs et les ruminants. D'autre part, dans un but de simplification, nous essayerons d'apprécier et de classer approximativement les 
TABLEAU $I$

Travaux sur rats.

\begin{tabular}{|c|c|c|c|}
\hline Produits & Valeur & Observations & Auteurs \\
\hline $\begin{array}{l}\text { Phosphates solubles : monopo- } \\
\text { tassique, disodique, ammo- } \\
\text { niacaux, phosphates d'urée. }\end{array}$ & $\mathrm{TH}$ & & CABEl, et al., 1950. \\
\hline $\begin{array}{l}\text { Phosphate tricalcique ( } \alpha \text { et } \beta \text { ) } \\
\text { et farine d'os. }\end{array}$ & $\begin{array}{c}\mathrm{B} \\
\mathrm{TB} \text { à B }\end{array}$ & & $\begin{array}{l}\text { REINACI et al., } 1952 . \\
\text { ELLIS et al., } 1945 .\end{array}$ \\
\hline Pyrophosphate acide de sodium. & $\begin{array}{l}\mathrm{TB} \\
\text { TB }\end{array}$ & & $\begin{array}{l}\text { ElLis et al., } 1945 . \\
\text { CABELL et al., } 1950 .\end{array}$ \\
\hline $\begin{array}{l}\text { Tripolyphosphate sodique, py- } \\
\text { rophosphate tétrasodique. }\end{array}$ & $\mathrm{TB}$ & & CABELL et al., 1950 \\
\hline$\gamma$ pyrophosphate. & $\mathrm{AB}$ & & ELLIS et al., 1945. \\
\hline B pyrophosphate. & $\mathbf{P}$ & & ELLIS et al., 1945. \\
\hline Métaphosphate insoluble. & $\begin{array}{l}\mathrm{P} \\
\mathrm{P} \\
\mathrm{M}\end{array}$ & . & $\begin{array}{l}\text { ELLIS et al., } 1945 . \\
\text { CABELL et al., } 1950 . \\
\text { FrasER et al., } 1943 .\end{array}$ \\
\hline Métaphosphate vitreux. & $\mathrm{TB}$ & Soluble dans l'eau. & CABliLr et al., 1950 \\
\hline Métapolyphosphate de $K$. & M & $\begin{array}{l}41 \text { à } 56 \% \text { absorbé mais peu } \\
\text { retenu. }\end{array}$ & LANG et al., $195 \overline{5}$ \\
\hline Hexamétaphosphate de Na. & $\mathrm{ML}$ & $\begin{array}{l}34 \text { à } 63 \% \text { absorbé et } 9,7 \% \\
\text { retenu dans les tissus. } \\
\text { Aussi bien que le bipotassique. } \\
\text { Rétention de } 38 \text { à } 43 \% .\end{array}$ & $\begin{array}{l}\text { LANG et al., } 1955 . \\
\text { DrMSZA et al., } 1959 .\end{array}$ \\
\hline $\begin{array}{l}\text { Phosphates naturels de Rhé- } \\
\text { nanie. }\end{array}$ & $\mathrm{AB}$ à $\mathrm{P}$ & Suivant la teneur en $\mathrm{F}$. & CABELL et al., 1950 \\
\hline Phosphate de roche fondu. & I13 & & Fraser et al., 1949. \\
\hline Phosphates de roche défluorés. & $\begin{array}{l}\mathrm{TH} \\
\mathrm{B} \text { à } \mathrm{M}\end{array}$ & $\begin{array}{l}\text { Suivant le traitement de cléfuo- } \\
\text { ration. }\end{array}$ & $\begin{array}{l}\text { Ellis et al., 19\%5. } \\
\text { BARRENTINE et al., 19\% }\end{array}$ \\
\hline $\begin{array}{l}\text { Phosphates défluorés "Elec- } \\
\text { trofos ". }\end{array}$ & $\mathrm{M}$ & $\begin{array}{l}\text { Avec des doses doubles donne } \\
\text { des résultats proches de } \\
\text { ceux obtenus par le phos- } \\
\text { phate bicalcique. }\end{array}$ & ReinaCi et al., 1950. \\
\hline Superphosphates défluorés. & B à M & & CABELl et al., 1950. \\
\hline $\begin{array}{l}\text { Superphosphates défluorés à } \\
1010^{\circ} \mathrm{C} \text {. }\end{array}$ & $\mathrm{B}$ & & lílis et al., 19'́. \\
\hline $\begin{array}{l}\text { Superphosphates défluorés à } \\
300^{\circ} \mathrm{C} \text {. }\end{array}$ & $\mathrm{Al3}$ à $\mathrm{M}$ & & ELI.IS et al., 1945. \\
\hline $\begin{array}{l}\text { Superphosphates défluorés à } \\
600 \text { et } 760^{\circ} \mathrm{C} \text {. }\end{array}$ & $\mathrm{I}$ & & ELLIS et al, 1945 \\
\hline Scories. & b̀̀ $\mathrm{AB}$ & & ELLIS et al., 1945. \\
\hline $\begin{array}{l}\text { "Ferro-phosphore " (résidu de } \\
\text { four électrique soluble dans } \\
\text { HCl à } 0,4 \% \text { ). }\end{array}$ & $\mathrm{P}$ & $\begin{array}{l}\text { Après addition de craie donne } \\
\text { de très bons résultats. }\end{array}$ & WiLliams et al., 1949. \\
\hline $\begin{array}{l}\text { Phosphates de roches naturels } \\
\text { alumino ferriques. }\end{array}$ & $\mathrm{M}$ & & $\begin{array}{l}\text { ELLIS et al., } 1945 . \\
\text { REINACH et al., } 1958\end{array}$ \\
\hline
\end{tabular}


TABLEAU 2

Travaux sur les volailles.

\begin{tabular}{|c|c|c|c|}
\hline Produits & Valeur & Observations & Auteurs \\
\hline I'hosp hate tricalcique ( $\alpha$ ou $\beta$ ). & $\begin{array}{c}\mathrm{AB} \\
\mathrm{TB} \\
\mathrm{TB} \\
\mathrm{AB} \text { à } \mathrm{M} \\
\mathrm{AB} \\
\mathrm{AB}\end{array}$ & $\begin{array}{l}\text { Inférieur à la poudre d'os. } \\
\text { Comme la poudre d'os. } \\
\text { La différence avec le phos" } \\
\text { phate bicalcique diminue } \\
\text { pour un haut niveau de P. }\end{array}$ & $\begin{array}{l}\text { MotzoK et al., } 1956 . \\
\text { SINGSEN et al., } 1946 . \\
\text { GILLIS et al., } 195 \% \\
\text { WILCOX et al., } 1955 . \\
\text { WILCOX et al., } 1954 . \\
\text { CREECH, } 1956 .\end{array}$ \\
\hline Phosphate monocalcique. & $\begin{array}{l}\mathrm{TB} \\
\mathrm{TB}\end{array}$ & & $\begin{array}{l}\text { WILcox et al., } 1954 . \\
\text { WILcox et al., } 1955 .\end{array}$ \\
\hline Phosphate monopotassique. & TB & & EDWARDS et al., 1958. \\
\hline Phosphate monosodique. & $\mathrm{TB}$ & Comme la poudre d'os. & SINGSEN et al., 1946. \\
\hline Phosphate bicalcique anhydre. & M & $\begin{array}{l}\text { Solubilité bien inférieure à } \\
\text { celle de la forme cristalline. }\end{array}$ & EDWARDS et al., 1958. \\
\hline Phosphates bicalciques. & $\mathrm{AB} \dot{\mathrm{a}} \mathrm{TB}$ & $\begin{array}{l}\text { Différences non expliquées entre } \\
\text { sources. }\end{array}$ & WiLCox et al., 1955 \\
\hline 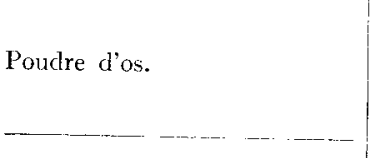 & $\begin{array}{c}\mathrm{B} \\
\mathrm{AB} \\
\mathrm{B} \\
\mathrm{TB}\end{array}$ & & $\begin{array}{l}\text { MILLER et al, } 1953 . \\
\text { WILCOX et al., } 1954 . \\
\text { SINGSEN et al., } 1946 . \\
\text { HotzoK et al., } 1956 .\end{array}$ \\
\hline 3 pyrophosphate. & $\begin{array}{l}\mathrm{P} \\
\mathrm{P}\end{array}$ & $\begin{array}{l}\text { Essais avec régimes très puri- } \\
\text { fiés. }\end{array}$ & $\begin{array}{l}\text { GILLIS et al., 195'. } \\
\text { BIRD et al., } 1945 .\end{array}$ \\
\hline Métaphosphate cristallisé. & $\begin{array}{l}\mathrm{P} \\
\mathrm{M} \\
\mathrm{M}\end{array}$ & & $\begin{array}{l}\text { GILLIS et al., 195\%. } \\
\text { MicCONNELL et al., } 1954 . \\
\text { MATTERSON et al., 19'5. }\end{array}$ \\
\hline $\begin{array}{l}\text { Métaphosphate vitreux (amor- } \\
\text { phe). }\end{array}$ & $\begin{array}{l}\mathrm{AB} \\
\mathrm{AB}\end{array}$ & $\begin{array}{l}\text { Comme le phosphate trical- } \\
\text { cique, mais résultat obtenu } \\
\text { avec ration de base riche } \\
\text { en } P \text {. }\end{array}$ & $\begin{array}{l}\text { BIRD et al., } 19 \% 3 . \\
\text { BIRD et al., } 1945 .\end{array}$ \\
\hline hosphate de roche brut. & $\begin{array}{c}\mathrm{M} \\
\mathrm{AB} \text { à } \\
\mathrm{M}^{\mathrm{M}} \\
\mathrm{M} \\
\mathrm{B}\end{array}$ & $\begin{array}{l}\text { Après l'âge de huit semaines } \\
\text { les résultats sont } \mathrm{AB} \text {. } \\
\text { Expliquent les bons résultats } \\
\text { obtenus par la richesse en } \\
\text { oligo-éléments. }\end{array}$ & $\begin{array}{l}\text { GLRRY et al., } 1947 . \\
\text { GILLIS et al., } 1954 . \\
\text { MCCONNELL et al., } 1944 . \\
\text { WiLCOX et al., } 195 \% \\
\text { MATTERSON et al., } 1945 .\end{array}$ \\
\hline hosphate de roche fondu. & $\begin{array}{c}\mathrm{TB} \\
\mathrm{B}\end{array}$ & & $\begin{array}{l}\text { MCCONNELL et al., } 1944 . \\
\text { MATTERSON et al., } 1945 .\end{array}$ \\
\hline uperphosphate. & $\mathrm{AB}$ & Assez riche en F. & GERRY et al., 1947. \\
\hline cories. & $\mathrm{AB}$ & & BIRD et al., 1945 \\
\hline
\end{tabular}


TABIEAC 2 (suite)

\begin{tabular}{|c|c|c|c|}
\hline Produits & Valeur & Observations & Auteurs \\
\hline Phosphate naturel (de Curaçao). & $\begin{array}{c}\mathrm{B} \\
\mathrm{AB} \\
\mathrm{M} \\
\mathrm{B}\end{array}$ & & $\begin{array}{l}\text { GiLLis et al, } 195 y^{\prime} . \\
\text { AMMERMAN, } 1956 . \\
\text { LiDWARIS et al., } 1958 . \\
\text { MILLER et al., } 1933 .\end{array}$ \\
\hline Phosphate argileux colloïdal. & $\begin{array}{l}\mathrm{M} \\
\mathrm{M} \\
\mathrm{M} \\
\mathrm{M} \\
\mathrm{a} \mathrm{Ab} \\
\mathrm{M} \\
\mathrm{Al} \\
\mathrm{M} \\
\mathrm{M} \\
\mathrm{M}\end{array}$ & 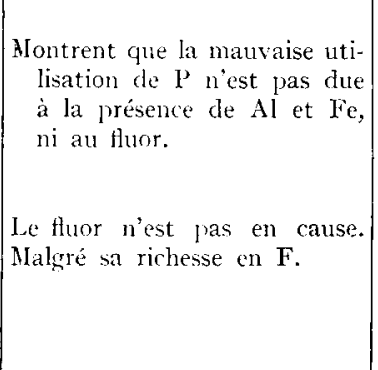 & 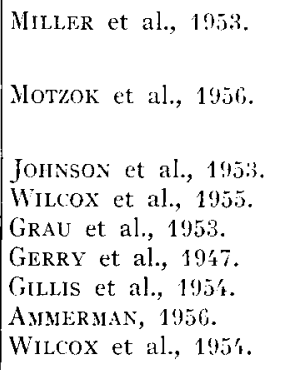 \\
\hline $\begin{array}{l}\text { Mélange de phosphate collö́- } \\
\text { dal et de phosphate mono- } \\
\text { potassique (à parties égales). }\end{array}$ & B & $\begin{array}{l}\text { Aussi bons résultats qu'avec } \\
\text { le phosphate monopotassique } \\
\text { seul. }\end{array}$ & УотzоK et al., 1956. \\
\hline l'hosphates detluorés. & $\begin{array}{ccc}\mathrm{B} \\
\mathrm{TB} \\
\mathrm{P} \\
\mathrm{TB} & \mathrm{a} & \mathrm{AB} \\
\mathrm{TB} & \mathrm{à} & \mathrm{B}\end{array}$ & Variáble suivant les sources. & 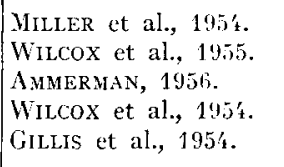 \\
\hline l'hosphates de roches clétluorés. & $A B$ & & BrRI et al., $1 \%$ ' 5. \\
\hline Superphosphates défluorés. & l' à $\mathrm{M}$ & $\begin{array}{l}\text { Sur plusieurs superphosphates, } \\
\text { un sur quatre est inutilisable. }\end{array}$ & BIRD et al, 1915 \\
\hline
\end{tabular}

efficacités biologiques des différents produits suivant cinq groupes ainsi définis : très bon ('T. B.), Bon (B), Assez bon (AB), médiocre (M) et pauvre (P). Les coefficients de digestibilité et de rétention ayant rarement été calculés dans les expériences effectuées, nous devrons bien entendu procéder par comparaison, en admettant que le phosphate bicalcique par exemple est très bien utilisé chez toutes les espèces. Ces appréciations qualitatives résultent de la confrontation aussi objective que possible de tous les résultats, mais ne possèdent cependant qu'une valeur relative.

Il est bien difficile de dégager de cette série de résultats, parfois contradictoires, un classement suivant les efficacités biologiques, ne tenant compte que des différences de caractéristiques ou de composition entre les phosphates, abstraction faite des différences entre les méthodes d'évaluation.

Toutefois, il apparaît déjà que 1'accord entre les auteurs scmble réalisé four ce qui concerne les orthophosphates de sodium, potassium, calcium et pour la farine d'os. Ces produits semblent être en général bien utilisés par tous les animaux. En revanche, les phosphates bruts, naturels ou traités, et les phosphates aluminoferriques, conduisent à des résultats très variables, qu'il n'est pas possible de généraliser. 
TABLEAU 3

Travaux sur les porcs.

\begin{tabular}{|c|c|c|c|}
\hline Produits & Valeur & Observations & Auteurs \\
\hline Phosphate monocalcique. & TB & & PLCXILEF, et al., 1958. \\
\hline $\begin{array}{l}\text { Acide phosphorique. Farine } \\
\text { d'os. }\end{array}$ & $\mathrm{B}$ & & PLCMLFE et al., 1958. \\
\hline Chloroapatite. & TB & $\begin{array}{l}\text { Coefficient d'utilisation diges- } \\
\text { tive de } 45 \% \text {. }\end{array}$ & ARRINGTON et al., 1959. \\
\hline Phosphate tricalcique naturel. & $M$ & $\begin{array}{l}\text { Non défluoré : le fluor semble } \\
\text { étre la cause des arcidents } \\
\text { observés. }\end{array}$ & Péro, l'tí. \\
\hline Phosphate naturel de Curayao & $\mathrm{AB}$ & & Pltwlef et al., 1958. \\
\hline $\begin{array}{l}\text { Phosphate naturel " argileux } \\
\text { collö̈dal ". }\end{array}$ & $\begin{array}{c}\mathrm{P} \\
\mathrm{AB} \\
\mathrm{B} \\
\mathrm{AB} \\
\mathrm{AB}\end{array}$ & $\begin{array}{l}\text { Rélonse variable suivant les } \\
\text { critères utilisés. } \\
\text { Ration de base trop riche en } \mathrm{P} \text {. } \\
\text { Coefficient d'utilisation diges- } \\
\text { tive de } 22 \% \text {. } \\
\text { Sans grosses perturbations, } \\
\text { mais vitesse de croissance } \\
\text { réduite. }\end{array}$ & 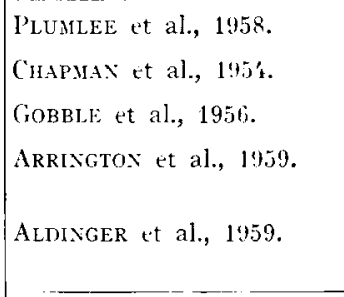 \\
\hline Phosphates naturels défluorés. & $\begin{array}{l}\mathrm{B} \\
\mathrm{B}\end{array}$ & $\begin{array}{l}28 \% \text { du phosphore ingéré a } \\
\text { eté retenu. }\end{array}$ & 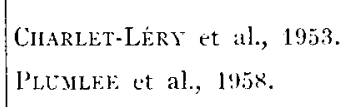 \\
\hline $\begin{array}{l}\text { Phospl hate alumino-ferrique na- } \\
\text { turel. }\end{array}$ & $\mathrm{P}$ & $\begin{array}{l}\text { Coefficient dutilisation diges- } \\
\text { tive de } 7 \% \text {. }\end{array}$ & Aldirgrer et al., 1959. \\
\hline
\end{tabular}

\section{IV. - DISCUSSION}

Pour essayer d'expliquer les contradictions, parfois apparentes, relevées dans les tableaux de résultats, nous reprendrons l'étude des influences respectives des méthodes et critères d'appréciation d'une part, et d'autre part de la nature même des phosphates étudiés.

\section{$\mathrm{I}^{\mathrm{O}}$ Influence des méthodes et critères d'appréciation de l'efficacité biologique des phosphates.}

Les animaux utilisés et leur mode d'alimentation. - Ians la présentation des résultats nous avons déjà éliminé l'influence de l'espèce en considérant que les résultats obtenus sur une espèce peuvent difficilement être généralisés aux autres. D'autre part, nous avons déjà insisté sur la variabilité des résultats obtenus en fonction des besoins en $\mathrm{P}$ des animaux utilisés. Ainsi, comme le soulignent Ammermix et a1. (I957) l'emploi d'animaux jeunes et à croissance rapide, préalablement soumis à un régime de déplétion en $P$, permet d'accentuer au maximum les différences entre plusieurs phosphates. Ceci explique les écarts observés par ces mêmes auteurs pour l'utilisation 
TABLEAU 4

Travaux sur ruminants

\begin{tabular}{|c|c|c|c|}
\hline Produits & Valeur & Observations & Auteurs \\
\hline $\begin{array}{l}\text { Phosphates bicalcique, mono- } \\
\text { calcique, tricalcique, mono- } \\
\text { sodique et disodique, farine } \\
\text { d'os. }\end{array}$ & $\mathrm{TB}$ & $\begin{array}{l}\text { Phosphates reconnus comme } \\
\text { étant très bien utilisés par } \\
\text { les ruminants. }\end{array}$ & Tous les auteurs. \\
\hline Acide phosphorique. & $\mathrm{TB}$ & & $\begin{array}{l}\text { Wisk et al., } 1958 . \\
\text { TILLMAN et al., } 19586 . \\
\text { RICIIARDSON et al., } 1955 . \\
\text { MENZIES et al., } 1955 . \\
\text { JARL, } 1953 .\end{array}$ \\
\hline $\begin{array}{l}\text { Métaphosphate vitreux (amor- } \\
\text { phe). }\end{array}$ & $\begin{array}{l}\mathrm{M} \text { à } \mathrm{AB} \\
\mathrm{AB}\end{array}$ & $\begin{array}{l}\text { Absorption normale mais } \\
\text { accroissement de l'excré- } \\
\text { tion fécale endogène. } \\
\text { Bien absorbé mais partielle- } \\
\text { ment réexcrété. }\end{array}$ & $\begin{array}{l}\text { Tillmian et al., } 1958 \mathrm{a} . \\
\text { AMMERMAN et al., } 1957 .\end{array}$ \\
\hline $\begin{array}{l}\beta \text { et } \gamma \text { métaphosphate cris- } \\
\text { talliś. }\end{array}$ & $\mathrm{P}$ & & AMMLRMAN et al., 1957. \\
\hline$\gamma$ pyrophosphate de calcium. & $P$ & $\begin{array}{l}\text { Partiellement absorbé puis ré- } \\
\text { excrété. De plus, accroît } \\
\text { l'excrétion enclogène de P. }\end{array}$ & AMMERMAN et al., 1957. \\
\hline Pyrophosphate acide de Na. & B & $\begin{array}{l}\text { Absorption étudiée à l'aide } \\
\text { de } 32 \mathrm{P} \text {. }\end{array}$ & THLLMAN et al., $1958 a$. \\
\hline $\begin{array}{l}\text { Phosphate naturel de roche } \\
\text { brut (de Curaçao). }\end{array}$ & $\begin{array}{l}\mathrm{B} \\
\mathrm{AB}\end{array}$ & $\begin{array}{l}\text { Résultats obtenus après pé- } \\
\text { riode de déplétion en P. } \\
\text { Digestibilité vraie de } 90 \% \\
\text { sur agneaux. } \\
\text { Sur veaux de } 15 \text { semaines avec } \\
\text { rations de base semi-purifiées. }\end{array}$ & $\begin{array}{l}\text { LONG et al., } 1957 . \\
\text { AMMERMAN, } 1956 . \\
\text { WENTWORTI et al., } 1960 .\end{array}$ \\
\hline Phosphate naturel de Curaçao. & $\begin{array}{l}\mathrm{B} \\
\mathrm{B}\end{array}$ & $\begin{array}{l}\text { Rétention de } 38 \% \text { sur agneaux. } \\
\text { Résultats obtenus sur tau- } \\
\text { reaux à faibles besoins. }\end{array}$ & $\begin{array}{l}\text { AMMERMAN et al., } 1957 . \\
\text { AMMERMAN et al., } 1957 .\end{array}$ \\
\hline Phosphate de roche brut. & $B$ & $\begin{array}{l}\text { Sur veaux. Le taux de F doit } \\
\text { etre surveillé. }\end{array}$ & ALtPOV, 1955. \\
\hline Phosphate argileux colloïdal. & $\begin{array}{l}\mathrm{M} \\
\mathrm{M} \\
\mathrm{B}\end{array}$ & $\begin{array}{l}\text { Sur moutons, avec }{ }^{32} \mathrm{P} \text {. CUD } \\
\text { réel de } 1 \dot{\%} \% \text { contre } 50 \% \\
\text { pour le bicalcique. } \\
\text { Le fuor n'est pas en cause } \\
\text { dans ce faible résultat. } \\
\text { La moitié du } \mathrm{P} \text { de la ration } \\
\text { est fournie par la ration de } \\
\text { base. } \\
\text { Sur agneaux, avec rétention } \\
\text { de } 13 \% \text {. } \\
\text { Sur veaux de } 15 \text { semaines et } \\
\text { ration de base semi-purifiée. } \\
\text { Sur tanreaux à faibles besoins. }\end{array}$ & $\begin{array}{l}\text { LOFGREEN, } 1960 . \\
\text { LONG et al., } 1956 . \\
\text { AMMERMAN et al., } 195 \% \text {. } \\
\text { AMMERMAN et al., } 1957 . \\
\text { WENTWORTH et al., } 1960 . \\
\text { AMMERMAN et al., } 1957 .\end{array}$ \\
\hline
\end{tabular}


TABLEAC 4 (stite)

\begin{tabular}{|c|c|c|c|}
\hline Produits & Valeur & Observations & Auteurs \\
\hline Phosphates défluorés. & $A B$ & $\begin{array}{l}\text { Sur taureaux dum an, provoque } \\
\text { mauvaise croissance, car seu- } \\
\text { lement la moitié du b' se } \\
\text { trouve sous forme ortho. } \\
\text { Traités par des acides pour } \\
\text { éviter la polymérisation. } \\
\text { Aussi bon que le phos]hate } \\
\text { monopotassique sur reaux, } \\
\text { digestibilité vraie de } 70 \% \\
\text { Digestibilité vraie de } 5 \text { ' \% sur } \\
\text { agneaux. } \\
\text { Sur veaux de } 15 \text { semaines et } \\
\text { ration de base semi-purifice. }\end{array}$ & 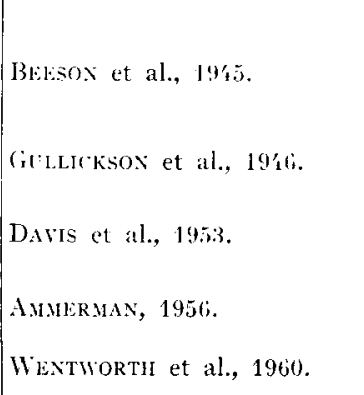 \\
\hline Phos] hates défluorśs. & $\begin{array}{l}\text { ll à } A B \\
B\end{array}$ & $\begin{array}{l}\text { La moitié de } P \text { fourni par la } \\
\text { ration de base. } \\
\text { Acidulés aprés la calcination. } \\
\text { Rétention de } 11 \text { o sur } \\
\text { agneaux. } \\
\text { Sur taureaux. }\end{array}$ & $\begin{array}{l}\text { AMMERMAN et al., } 1951 . \\
\text { AMMERMAN et al., } 1957 . \\
\text { AMMERMAN et al., } 1957 .\end{array}$ \\
\hline $\begin{array}{l}\text { Phosphate re roche alumino- } \\
\text { ferrique (sans Ca, avec } 16 \% \\
\left.\mathrm{Al}_{2} \mathrm{O}_{3} \text { et } 7 \text { a } 15 \% \mathrm{Fe}_{2} \mathrm{O}_{3}\right)\end{array}$ & P & $\begin{array}{l}\text { Sur brebis, par déplétion préa- } \\
\text { lable, donne des signes très } \\
\text { nets de carence en P. Bilan } \\
\text { de } \mathrm{P} \text { variant de }-4 \text { à } 8 \% \text {, } \\
\text { contre } 20^{\circ} \text { jour la farine d'os. }\end{array}$ & REIxtril et al, 19586 . \\
\hline $\begin{array}{c}\text { Phosphate alumino-calcique. } \\
\text { (ave ti } \% \mathrm{Fe} \text { ) }\end{array}$ &.$A B$ & $\begin{array}{l}\text { Sur agneaux de } 7 \text { mois } 1 / 2 \text { par } \\
\text { déplétion préalable et ration } \\
\text { semi-puritiée. Rétention de } \\
22,7 \% \text { contre } 31 \% \text { aver le } \\
\text { monosodique. }\end{array}$ & Zlitter et al., 1960. \\
\hline
\end{tabular}

du phosphore dans les mêmes produits, d'une part par des agneaux en pleine croissance, et d'autre part par des taureaux à l'entretien. Cependant, plusieurs travaux sur les poulets (BIRD et al. I943, GERRY et al. I947) semblent montrer que les anima11x âgés seraient moins sensibles aux différences de digestibilité du $\mathrm{P}$ de plusieurs sources. Ainsi, le phosphate de roche étudié, mal utilisé avant l'âge de 8 semaines, donne de bons résultats après I 6 semaines (GERRY et al. I947). I1 semble néanmoins difficile de conclure si les bons résultats obtenus chez des sujets adultes sont dûs à une meilleure aptitude de ces animaux à utiliser du phosphore peu assimilable, ou plutôt simplement au fait que leurs besoins en $\mathrm{P}$ sont faibles.

La proportion du $\mathrm{P}$ apporté par le complément étudié par rapport au phosphore total de la ration est l'une des principales causes des controverses entre les auteurs. Ainsi TrLER (I948) critique la méthode des régimes semi-purifiés, consistant à fournir, pour les comparaisons de phosphates, une ration de base très pauvre en $P$. Dans ces conditions, les différences obtenues entre deux sources de $\mathrm{P}$ sont certainement accentuées. Toutefois, si ces résultats présentent un certain intérêt biochimique et physiologique, ils sont de peu d'importance pour l'éleveur ; en effet, si l'utilisation du $\mathrm{P}$ du complément varie avec le taux de $\mathrm{P}$ de la ration de base, il importe d'effectuer les comparaisons en employant des rations de base courantes. Cependant, il est bien 
évident que si la ration de base fournit une quantité de P trop proche des besoins minima de l'animal, et si les critères d'appréciation ne considèrent que l'utilisation globale du $\mathrm{P}$ total de la ration, il ne faudra pas s'attendre à des différences entre sources de $P$, le supplément n'étant pas nécessaire. Ceci explique sans doute les résultats de GoBbre et al. (I956) sur le phosphate argileux colloïdal pour les porcs, en contradiction avec ceux des autres auteurs.

C'est pourquoi PlumLeE et al. (I958) recommandent, pour de telles comparaisons, d'employer des rations de base suffisamment pauvres en $P$, tout en fournissant par chacun des compléments une quantité suffisante de $\mathrm{P}$ pour couvrir les besoins minima de l'animal.

La technique utilisée par LOFGREEN (I960) permet de pallier au mieux les inconvénients précédents et de fournir les résultats les plus valables sur 1'utilisation propre $\mathrm{du} \mathrm{P}$ du phosphate. Cet auteur utilise à cet effet des rations de base relativement pauvres en $\mathrm{P}$ (le supplément double l'apport de $\mathrm{P}$ dans la ration) et emploie la méthode de dilution isotopique d'une part avec la ration de base seule, d'autre part avec la ration de base plus le supplément. Ainsi, connaissant l'utilisation globale de $\mathrm{P}$ dans les deux cas, il peut en déduire la digestibilité vraie du $\mathrm{P}$ fourni par le supplément.

Notons également que des taux élevés de suppléments phosphatés peuvent dans certains cas (BIRD et al. I945) provoquer un effet dépressif mal expliqué, et donner ainsi des résultats inférieurs à ceux obtenus par des ingestions modérées. D'après Til.mman et al. (I959) le taux de $\mathrm{P}$ dans la ration n'influe que faiblement sur la digestibilité réelle et sur l'excrétion fécale endogène de $P$. L'effet dépressif d'un excès de certains phosphates serait done dî̀ à l'influence d'autres constituants de la ration.

Les critères d'appréciation. - Les résultats obtenus dans l'appréciation des besoins en $P$ des animaux varient suivant les critères utilisés. Ainsi 'TrL.max et al. (I959) trouvent qu'un apport de $4 \mathrm{~g}$ de $\mathrm{P}$ par Ioo $\mathrm{kg}$ de poids vif est insuffisant pour couvrir les besoins de jeunes taureaux, si l'on considère comme critères les gains de poids et les coefficients de digestibilité et de rétention du phosphore; en revanche, pour la croissance de l'os et le maintien du taux de $\mathrm{P}$ sérique, cet apport semble suffisant. Donc, en comparant l'utilisation du $\mathrm{P}$ de deux phosphates, les différences de croissance de l'os et de taux de $\mathrm{P}$ du sérum apparaîtront plus difficilement que les modifications de la courbe de croissance. D'autre part, les différences de gains de poids ne se manifestent qu'après 1'apparition de différences très nettes dans la structure et la composition des os, et, qui mieux est, la teneur en cendres du fénur et sa résistance peuvent varier en sens inverse (CHApman et al. I954). Ceci pourrait s'expliquer, selon ces auteurs, par une influence spécifique de certains phosphates sur la structure intime de l'os, par des modifications physiques subies par les cristaux d'hydroxyapatite.

Comme les besoins pour le maintien du taux de $\mathrm{P}$ sérique sont les plus faibles, la mauvaise utilisation du $\mathrm{P}$ d'un phosphate sera donc plus difficile à déceler si l'on s'appuie uniquement sur les modifications de la teneur en $\mathrm{P}$ du sang. Il existe donc une hiérarchie certaine entre les critères et une chronologie dans l'apparition des symptômes.

Notons enfin que le test du P sérique donne parfois une mauvaise indication de l'utilisation du phosphore. En effet, des travaux effectués sur des métaphosphates 
purs (Tillman et al. I958) et sur le $\gamma$ pyrophosphate (Ammerman et al. I957), montrent qu'une partie importante du $\mathrm{P}$ absorbé, permettant de maintenir un taux de $\mathrm{P}$ sérique normal, est ensuite réexcrétée dans les fèces. L'accroissement de l'excrétion endogène fécale entraîne donc une mauvaise rétention du phosphore, bien que son absorption intestinale soit normale.

Malgré les difficultés d'interprétation qui résultent d'une telle diversité de méthodes et de critères d'appréciation, il est cependant possible de dégager des différences entre certaines formes de phosphates.

\section{$2^{\circ}$ Influence propre de la nature du phosphate.}

De nombreux facteurs, propres au produit même, influent sur l'utilisation du $\mathrm{P}$ par les animaux.

La finesse des particules semble avoir une influence négligeable sur 1'assimilabilité du phosphore. Des essais sur poussins (Gillis et al. I95I), employant cinq degrés de môtuture pour le phosphate tricalcique, montrent que seul le phosphate le plus grossier est légèrement moins bien utilisé que les autres. Toutefois, les produits finement divisés et amorphes sont en général plus solubles que les produits grossiers et bien cristallisés (HIL, et al. I945).

La forme chimique et le degré de polymérisation semblent exercer une influence prépondérante sur la disponibilité du $\mathrm{P}$ pour les animaux. Ainsi, en règle générale, et quelle que soit l'espèce animale, les orthophosphates purs (calciques, sodiques, potassiques) sont très bien utilisés. En revanche, les métaphosphates et les formes polymérisées (polyphosphates, en particulier les pyrophosphates) sont en général moins utilisables. Nous avons déjà signalé que le phosphore des méta et pyrophosphates, bien que parfois absorbé sous cette forme, était ensuite réexcrété dans le tractus digestif. La faible utilisation du $\mathrm{P}$ de ces phosphates serait donc due, outre une faible digestibilité, à une mauvaise rétention du $\mathrm{P}$ absorbé. C'est ainsi que AMmerman et al. (I957) trouvent, dans des essais sur agneaux, des coefficients de rétention du $\mathrm{P}$ variant de $26 \mathrm{p}$. Ioo pour un orthophosphate (phosphate monocalcique), à o p. Ios pour un métaphosphate (métaphosphate de calcium vitreux) et à - 2I p. Ioo pour un pyrophosphate ( $\gamma$ pyrophosphate de Ca). De plus, en calculant les coefficients de digestibilité réelle du $\mathrm{P}$ du complément (le $\mathrm{P}$ de la ration de base étant exclu), ils trouvent respectivement $40 \mathrm{p}$. IOO, IO $\mathrm{p}$. IoO et $-22 \mathrm{p}$. IOO pour les formes ortho, méta vitreux et pyro, en adoptant un taux normal d'excrétion endogène du phosphore (calculé d'après un essai préliminaire). Si le pyrophosphate était totalement inassimilable, sans accroître l'excrétion endogène de $\mathrm{P}$ par l'agneau, on aurait une absorption vraie de o au lieu de -22 p. Ioo; donc le pyrophosphate accroît le phosphore métabolique des fèces, ce qui rend l'utilisation de ces formes de phosphates nocive pour l'animal.

Des essais sur rats, utilisant des formes complexes de polyphosphates, semblent toutefois protuver qu'une partie du $\mathbf{P}$ fourni serait hydrolysée dans l'intestin et absorbée sous forme ortho (SCHREIER et al. I955, LANG et al. I955).

Enfin, ces derniers auteurs trouvent que 1'hexamétaphosphate est mal utilisé, tandis que Dymsza et al. (I959), également sur rats, trouvent une rétention de 38 à 43 p. Ioo pour l'hexamétaphosphate de Na.

La forme cristalline influe considérablement sur la digestibilité du P au sein de chacun des groupes ortho, méta ou pyrophosphates. Ainsi les différences observées 
entre les phosphates bicalciques anhydre et hydraté seraient vraisemblablement dues à une solubilité moindre de la forme anhydre (EDwards et al. 1958). Tous les essais effectués sur le phosphate tricalcique montrent que les formes $\alpha$ et $\beta$, c'est-à-dire hydratée et anhydre ( $\alpha$ désignant également la forme cristalline stable au point de fusion) sont également utilisées par les animaux. En revanche, la forme $\gamma$ du pyro-

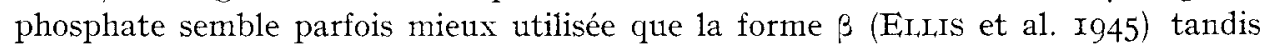
que, par rapport au métaphosphate amorphe, soluble et généralement assimilable, les deux formes cristallines $\alpha$ et $\beta$ sont de très mauvaises sources de $P$ pour les animaux. Cette moindre digestibilité de certains phosphates cristallisés s'expliquerait par la formation dans l'intestin de polymères de longueur variable, gênant l'absorption (HENDRICKS, I944).

L'influence du traitement subi par les phosphates, en particulier lors de la défluoration, sur la disponibilité du $\mathrm{P}$, résulte donc des modifications éventuelles dans leur degré de polymérisation ou dans leur forme allotropique. Ceci explique parfaitement les différences considérables qui existent entre les efficacités biologiques de plusieurs superphosphates ou phosphates naturels défluorés de même origine (BARRENTINE et al. I944, Reinach et al. I950, Eiriss et al. I945, Almerman et al. I957, Wilcox I954, Gillis et al. I954, Bird et al. I945). Ces différences seraient donc dues à l'apparition, au cours du traitement thermique, de formes relativement insolubles de méta ou de pyrophosphates.

Des essais ont été effectués sur plusieurs phosphates défluorés à des températures variables. Ainsi, d'après les travaux de HrLL et al. (I945) sur des phosphates de calcium, la solubilité du $\mathrm{P}$ augmente considérablement de $600^{\circ} \mathrm{C}$ à I $000^{\circ} \mathrm{C}$. Ceci correspond au passage du stade méta au stade pyro, puis à la forme tricalcique. Au-delà de $\mathrm{I} 000^{\circ} \mathrm{C}$ il se forme de l'apatite. Eiris et al. (I945) ont comparé, dans des essais sur rats, l'efficacité biologique de plusieurs superphosphates défluorés à des températures variables: $300^{\circ} \mathrm{C}, 600^{\circ} \mathrm{C}, 760^{\circ} \mathrm{C}$, et $\mathrm{I} 010^{\circ} \mathrm{C}$.

Seuls les phosphates défluorés à I OIO ${ }^{\circ} \mathrm{C}$ donnent de bons résultats sur rats Le traitement à $300^{\circ} \mathrm{C}$ donne des résultats moyens, tandis que les traitements à $600^{\circ} \mathrm{C}$ et à $760^{\circ} \mathrm{C}$ conduisent à la formation de phosphates inutilisables, sans doute à cause de la présence abondante des formes intermédiaires méta et py ro insolubles.

I1 importe donc, semble-t-il, de veiller tout particulièrement au choix des températures employées pour les traitements thermiques subis par des phosphates destinés aux animaux, ou d'associer à la calcination un traitement acide approprié permettant d'éviter la polymérisation (Gullickson et al. 1946, Ammerman et al. I957).

Les autres constituants $d u$ phosphate influent également sur l'utilisation du $\mathrm{P}$ par les animaux. Nous avons déjà discuté l'importance attribuée au rapport phosphocalcique et à l'excès de calcium dans la ration. Toutefois, sachant que le premier objectif d'une alimentation minérale rationnelle est d'assurer un apport convenable de $\mathrm{P}$ et de $\mathrm{Ca}$, nous ne tiendrons pas compte du déséquilibre phosphocalcique de certains phosphates, puisqu'il peut être corrigé par d'autres constituants de la ration.

Le problème du fluor est également bien connu, et nous savons qu'il intervient par sa toxicité propre. C'est pourquoi il est nécessaire de défluorer, avec les risques d'insolubilisation du phosphore que cela comporte, les phosphates trop riches en fluor (teneur en $\mathrm{F}$ supérieure à 2 p. IOo). 
L'influence souvent néfaste d'autres métaux, tels que le fer, l'aluminium et le zinc est en revanche moins connue. Des essais sur agneaux (Thompson et a1. I959) recevant du sulfate de zinc à des taux de 0,5 et I p. Ioo de la ration, montrent que les taux élevés de $Z n$ diminuent la rétention nette de $P$ sans influer sur les pertes endogènes. Donc des quantités importantes de $\mathrm{Zn}$ diminueraient l'absorption $\mathrm{du} P$ de la ration.

Buttnefr et al. (I959) montrent également (qu'un excès de fer dans le régime contribue à la formation de phosphates insolubles au niveau de l'absorption, et nuit ainsi à l'utilisation du fer et du phosphore. Cependant, en ajoutant de l'oxyde de fer à du phosphate monocalcique, MotzoK et al. (1956) n'observent pas d'effet dépressif sur poulets; en fait, l'oxyde de fer étant insoluble, il ne f: $€$ ut se combiner at phosphore pour former des phosphates.

De très nombreux travaux sur petits mammifères (STREET I942, JONES I938, Mackenzie r931, Cox et a1. I931, Mc Collum et al. I928) et sur poulets (Deobald et a1. I935, Cox et a1. I93I) prouvent que des quantités importantes d'aluminium, en particulier sous forme de sulfate, nuisent à l'utilisation digestive du phosphore. En effet, 1'Al étant pratiquement inassimilable, son effet néfaste se situerait au niveau de l'absorption. I)es travaux récents sur agneaux ('Tномpsox et al. I959) donnent des résultats contraires : en distribuant des rations contenant du sulfate d'aluminium à des taux de 0,5 et I p. Ioo, ces auteurs n'observent qu'un effet dépressif négligeable sur la rétention nette de $P$, sans modification de l'excrétion fécale $\in$ ndogène. Pour ces mêmes teneurs en $\mathrm{Al}$ la digestibilité réelle de $\mathrm{P}$ était de 77 et 80 p. Ioo au lieu de 88 p. Ioo.

Il semble que le comportement du fer et de l'aluminium vis-à-vis du phosphore varie selon l'espèce, monogastrique ou ruminant. En effet, STRUTHERs et al. (I950) ont montré que la présence d'anions organiques modifieles conditions de précipitation des phosphates de fer et d'aluminium. Le phosphate d'Al précipite rormalement dans des conditions de $\mathrm{pH}$ variant de 4 à 9 . Toutefois, en présence d'anions organiques, et en particulier d'acides organiques très abondants dans le rumen, l'aluminium est complexé et la précipitation en phosphate ne s'effectue pas. Cette hypothèse paraît valable, et en fait, aucun travail expérimental ne prouve l'effet néfaste de Al sur le métabolisme du phosphore chez les ruminants.

Cas particulier des phosphates alumino-ferriques et des phosphates naturels argileux ou colloïdaux. - De nombreux travaux, précédemment cités, montrent la mauvaise utilisation par les animaux de certains phosphates contenant des quantités importantes de Fe et d'Al. La plupart des auteurs attribuent la cause de cette mauvaise utilisation à la présence de ces éléments étrangers. En fait, même dans le phosphate colloïdal, $\mathrm{Al}$ et Fe se trouvent en quantités suffisantes pour combiner tout le $\mathrm{P}$ sous forme de phosphates insolubles. Toutefois, la majente partie de ces éléments se trouve sotus forme d'oxydes insolubles ne pouvant se combiner au phosphore (AMmERMAN et al. I957). Motzor et al. (I956) montrent que du phosphate monocalcique surchargé d'oxydes de fer et d'aluminium ne donne pas les mênes effets que le phosphate colloïda1.

If a plupart des essais effectués sur les phosphates contenant du Fe ou de l'A1 concernent les monogastriques, pour lesquels, éventuellement, le métabolisme de $P$ serait perturbé par des quantités importantes de Fe et Al. Pour ce qui concerne les ruminants, seuls REIXACH et al. (I955) travaillant sur brebis, constatent un effet 
dépressif marqué des phosphates alumino-ferriques. Compte tenu des observations précédentes il semble que la cause de cette mauvaise utilisation de $\mathrm{P}$ devrait sans doute être attribuée à la présence de formes intermédiaires insolubles (méta ou pyro) et non à la présence de Fe et A1. De même, ZELTER et al. (I960), sur agneaux sevrés de 7 mois et demi, trouvent que le $\mathrm{P}$ du phosphate alumino-calcique employé est moins bien retenu que le $\mathrm{P}$ du phosphate monosodique (rétentions de $22,7 \mathrm{p}$. Ioo et 34,0 p. Ioo respectivement). Sa digestibilité est également inférieure et, après déplétion, il ne peut assurer un retour normal du taux de $\mathrm{P}$ sérique. D'autres travaux (Gasnier I955, RANDorN I955) sur lapins et rats montrent que le phosphore d'un phosphate alumino-ferrique est parfaitement utilisé par ces animaux. Selon BARUAH et al. (r959) l'addition de lactose (environ Io p. Ioo de la ration) augmente considérablement la digestibilité du $\mathrm{P}$ du phosphate colloïdal chez les volailles.

Signalons enfin que l'aluminium diminuerait la toxicité du fluor pour les ruminants (Thомеsox et al. I959, HobBs et al. I954).

\section{V - MÉTHODES D'ÉVALUATION IN VITRO DE L'EFFICACITÉ BIOLOGIQUE DES PHOSPHATES}

Les expériences de digestibilité sur animaux étant très longues et coûteuses, certains auteurs ont essayé d'apprécier l'efficacité biologique des phosphates au moyen de tests de solubilité, par analogie avec la détermination du phosphore assimilable dans les engrais. Notons dès à présent que la méthode au citrate d'ammonium utilisée pour les engrais ne convient pas pour estimer le phosphore assimilable pour les animaux (HAwK, I955).

Les tests les plus étudiés, et qui semblent donner des résultats valables, sont basés sur la solubilité du P dans l'acide chlorhydrique dilué (en général o,4 p. IOo) et dans l'acide citrique. Dans ces deux solvants, la solubilité des composés purs décroît de la forme ortho à la forme méta, la moins soluble, la solubilité de la forme pyro étant intermédiaire (HrL, et al. I945). En séparant les phosphates en deux grands groupes selon les résultats de digestibilité obtenus sur rats - (très bon et bon d'une part, médiocre et pauvre d'autre part) on peut constater que la limite de solubilité de $\sigma_{5} \mathrm{p}$. Ioo dans $\mathrm{HCl}$ à $0,4 \mathrm{p}$. Ioo permet de répartir dans ces mêmes groupes 75 p. Ioo des phosphates courants (EII,Is et al. I945. CABELI, et al. I950). Les produits insolubles dans $\mathrm{HCl}$ à $0,4 \mathrm{p}$. Ioo sont en général inutilisables comme sources de $\mathrm{P}$ pour les animaux. Bird et al. (I945) trouvent également un bon parallélisme entre les digestibilités obtenues pour plusieurs phosphates sur poulets, et leur solubilité à $3^{\circ} \mathrm{C}$ dans $\mathrm{HCl}$ à $0,25 \mathrm{p}$. roo.

'Toutefois, ces méthodes ne constituent qu'un moyen très grossier d'apprécier la valeur d'un phosphate et l'accord obtenu n'est pas parfait.

Les essais in vitro utilisant la technique du rumen artificiel semblent donner des résultats plus valables. Ainsi Raux et al. (1956) trouvent un excellent accord entre plusieurs séries de résultats obtenus au moyen du rumen artificiel et par des essais sur bovins et ovins. ANDERson et al. (1956), utilisant la même technique, trouvent également pour plusieurs phosphates des digestihilités en accord avec celles obtenues sur agneaux par Ammermax et al. (I957). 


\section{CONCLUSIONS}

L'appréciation de la valeur d'un phosphate comme source de phosphore pour les animaux, que ce soit au moyen de tests de solubilité dans des solvants appropriés ou par des essais comparatifs sur animaux, est en général délicate et peu précise. Ceci explique les nombreuses contradictions entre les travaux concernant 1'utilisation des phosphates.

Les sels purs, sources classiques de phosphore, comme les orthophosphates calciques, sodiques ou potassiques, semblent tous équivalents quelle que soit l'espèce animale. En revanche les phosphates naturels, bruts ou défluorés, et certains phosphates alumino-ferriques, sont plus ou moins bien utilisés par les animaux et en particulier par les monogastriques. Il convient donc, semble-t-il, de les employer avec prudence, en particulier dans les composés minéraux destinés aux volailles et aux porcs.

$$
\text { Rȩ̣ı en juillet } 1961 .
$$

\section{SUMMARY}

COMPARATIVE VALUE OF MINERAL PHOSPHATES AS SOURCES OF PHOSPHORUS FOR ANIMALS

The utilization by animals of phosphorus from phosphates may vary according to various factors inherent to the animal, to feeding habits and to the product itself. It is very difficult to estimate, from a choice of convenient criteriums, the nutritional value of a phosphate.

This study collects the main results concerning the comparative value of mineral phosphates as sources of phosphorus for animals. The many apparent contradictions can often be explained by differences between the means and methods applied for the estimation of the value of the product.

However, undoubtedly, there exists differences between phosphates which can be attributed only to the nature of the product : fineness of particles, chemical structure, rate of polymerisation, crystal structure, processing applied before use and other constituents of the phosphate. The case of phosphates containing a large amount of iron and aluminium has been particular by studied.

It is also possible to estimate approximatively the biological efficiency of a phosphate by in vitro methods.

If the pure orthophosphates, classical sources of phosphorus, are always well utilized by animals, on the contrary the efficiency of the natural phosphates, raw or defluorinated, varies much, while the alumino-ferric phosphates are generally the most badly utilized especially by the monogastrics.

\section{RÉFÉRENCES BIBLIOGRAPHIQUES}

Aldinger S. M., Speer V. C., Ashion G. C., Hays V. W., Catron D. V., i959. Effect of feeding different combinations of soft phosphate with colloidal clay and dicalcium phosphate with and without added fluorine for growing-finishing swine. J. anim. Sci., 18, 555-560.

Alipov V. V., 1955. Rock phosphates for animal feeding. Zhivotnovodstvo, 11, 98-100.

Ammerman C. B., Neumane A. L., Forbes R. M., Norton H. W., i954. Utilization of phosphorus from various inorganic sources by steers. J. anim. Sci., 13, 974 (abstr.).

AmMERMan C. B., I956. Utilization of inorganic phosphates by ruminants. Ph. D. Thesis. University of Illinois.

Ammerman C. B., Forbes R. M., Garrigus U. S., Neumann A. L., Norton H. W., Hatfield E. E., 1957. Kuminant utilization of inorganic phosphates. J. anim. Sci., 16, 796-8ro.

Anderson R., Cineng E., Burrougis W., I956. A laboratory technique for measuring phosphorus availability of feed supplements fed to ruminants. J. anim. Sci., 15, 489-495.

Arrington L. R., Ammerman C. B., Davis G. K., r959. Measurement of phosphorus utilization by swine by an isotope technique. J. anim. Sci., 18, I 503 (abstr.). 
Barrentine B. F., MaYnard L. A., Loosi J. K., i944. The availability of the calcium and phosphorus of defluorinated rock phosphate for the rat. J. Nutr., 27, 35-42.

Baruah J. N., Davies R. E., Reid B. L., Covch J. R., i959. Effect of lactose on the utilization of phosphorus from colloïdal phosphate by chicks. Proc. Soc. Exper. Biol. Med., 102, 722-725.

Beeson W. M., Hickman C. W., Bolin D. W., Johnson R. F., Rinehart E. F., i945. Phosphorus supplements for steers compared. Idaho agr. Exp. Sta. Bull. 240.

Bird H. R., CASkEy C. D., I943. Amorphous calcium metaphosphate as a phosphorus supplement for chicks. Poult? Sci. 22, 333-334.

Bird H. R., Mattixgly T. P., 'itues H. W., Hammond J. C., Kellogg W. L., Clark T. B., Weakley C. E., VAN LANDINGIIAM A. H., I945. Nutritive evaluation of defluorinated phosphates and other phosphorus supplements. J. Ass. Off. Agric. Chent., 28, i i 8-129.

Brocinart M., ig6r. Communication personnelle.

Buttner W., Muhler J. C., 1950. Liffect of dietary iron on phosphate metabolism. Proc. Soc. Exper. Biol. Med., 100, $40-442$.

Cabell C. A., Earle I. P., Kauffain W. R., Ellis X. R., ig5o. Nutritive evaluation of defluorinated phosphates and other phosphorus supplements. IV. Further utilization experiments with rats. I95o. J. Ass. Off. Agric. Chem., 33, 448-456.

Causeret J., Hegot D., I958. Influence du taux calcique du régime alimentaire sur l'utilisation physiologique du calcium de divers sels. II. Étude comparative du carbonate de calcium et du phosphate tricalcique. Ann. Zootech., 7 , 79-87.

Chapman H. L., Kastelic J., Ashton G. C., Catros D. V., 1954. A comparison of phosphorus from different sources for growing finishing swine. J. anim. Sct., 14, 1073-1085.

Charlet-lery G., François A. C., Leroy A. M., ig53. Utilisation digestive et rétention par le porc du phosphore et du calcium d'un phosphate naturel défluoré. Ann. Zootech., 2, 285-301.

Combs G. E., Vandepopulieke J. M., Wallace H. D., Koger M., ig6o. Phosphorus levels and calcium phosphorus ratios for young and growing finishing swine. J.anim. Sci., 19, 1255, (Abstr.).

Cox G. L., Donds M. I., Wigman H. B., Murphy F. J., I93I. The effects of high doses of aluminium and iron on phosphorus metabolism. J. Biol. Chem., 92, xi.

Creech B. G., Reid B. L., Covch J. R., 1956. Evaluation of a dicalcium phosphate supplement as a source of phosphorus for chicks. 1 . Comparison of dicalcium and tricalcium phosphates as a source of phosphorus in chick and poult rations. Poultry Sci., 35, 654-658.

Davis G. K., Arrivgton L. R., Outler J. C., i953. Availability of phosphorus in defluorinated phosphate fed to cattle after activation in the pile. J. anim. Sci., 12, 913 (abstr.).

DEobald H. J., Elvehjem C. A., I935. The effects of feeding high amounts of soluble iron and aluminium salts. Am. J. Physiol., 121, I 18.

Dowe T. W., Matsushima J., ARThaud V. H., I957. The effects of adequate and excessive calcium when fed with adequate phosphorus in growing rations for beef calves. J. anim. Sci, 16, 81 r-82 I.

Dymsza H. A., Reussner G. Jr, Thiessen R. Jr, 1959. Effect of normal and high intakes of orthophosphate and metaphosphate in rats. J. Nutrit., 69, 4I9-428.

Edwards H. II., Youvg R. J., Gillis M. B., I958. Phosphate availability studies with the ash of unidentified growth factor supplements. J. Nutr., 65, 305-3I6.

lillis N. R., Cabell C. A., Elmslie W. P., Fraps G.S., Phillips P. H., Willams D. E., 1945. Nutritive evaluation of defluorinated phosphates and other phosphorus supplements. III. Utilization experiments with rats. J. Ass. Off. Agric. Chem., 28, I $29-14^{2}$.

Fraser H. F., Hoppe T. C., Sullivan J. H., Smitil E. R., I943. Phosphate fertilizers as possible source of phosphorus for animals. Ind. Eng. Chem., 35, 1087-1090.

Gasnier A., 1955. Travaux non publiés du laboratoire de l'Institut professionnel de recherches et de contrôle de l'alimentation animale. Paris.

Gerry R. W., Carrick C. W., Roberts R. E., Havge S. M., i947. Phosphate supplements of different fluorine content as sources of phosphorus for chickens. Poullyy Sci., 26, 323-334.

Gillis M. B., Norris L. C., Heuser G. F., i95r. The influence of particle size on the utilization of phosphates by the chick. Poultry Sci, 30, 396-398.

Gillis M. B., Norris L. C., Heuser G. F., r954. Studies on the biological value of inorganic phosphates. J. Nutr., 52, II5-I25.

Gobble J. C., Miller R. C., Sherritt G. W., Dunne H. W., i956. Soft phosphate with colloïdal clay as a source of phosphorus for growing and fattening swine. Pennsylvania. Agr. Exp. Sta. Bull, 609.

Grau C. R., Zweigart P. A., I953. Phosphatic clay as a phosphorus source for chicks. Poultry Sci., 32, 500-503.

Gullickson T. W., OLson F. C., I946. Defluorinated rock phosphate as a phosphorus supplement to the rations of dairy cattle. J. Dairy Sci., 29, 31 7-320.

Hansard S. L., Plumlee M. P., I954. Effects of dietary calcium and phosphorus levels upon the physiological behavior of calcium and phosphorus in the rat. $7 . N u t r ., 54,17-3 \mathrm{I}$.

Harris R. S., I959. Discussion du rapport présenté par Verueulen C. W. : Effects of high calcium intake on urine in mammals. Feder. Proc., 18, rog6-1 102.

HARRISON H. E., 1959. Factors influencing calcium absorption. Feder. Proc., 18, 1085-1092.

HaWk P. B., 1955. Digestibility of degelatined bone meal. Chem. Ztg., 79, 245*246.

Hendricks S. B., 1944. Polymer chemistry of silicates, borates and phosphates. J. Wash. Acat. Sci, 34, $24 \mathrm{I}$, 


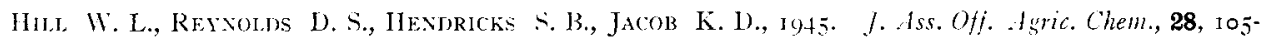
Irs.

Hobbs C. S., Mookman R. P., (iriffiti J. M., West J. L., Merriman G. M., Hansard S. I., Chamberlaix C. C., I954. Fluorosis in cattle and sheep. Tenn. Igric. Exp. Sla. Bull., 235.

JARL F., 1953. Mineral metabolism in dairy cows. Kangl. Lantbracksh. Ann., 20. 151-203.

Jomsson E. I., PHIIfIs's R. E., 195.3. T tilization of soft phosphate with collödal clay. Poultry Sci., 32,907 .

JoNes J. H., 1938. The metabolism of calciun and phosphorus as intluenced by the addition of salts of metals which form insoluble phosphates. Amer. J. Physiol., 124, 230.

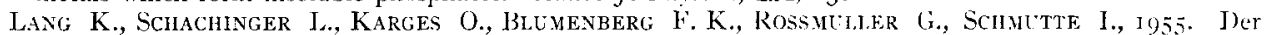
Stoffwechsel von Polyphosphaten. Biochem. Ztschr., 327, I I 8- I 25.

LitTlejohn A. I., LEWIs G., I960. Symposium on disorders of cilcium metabolisn. Experimental studies of the relationship between the calcium-phosphorus ratio of the diet and fertility in heifers : a preliminary report. Veter. Rec., 72, II37-II44.

LOFGREEN G. P., r960. The availability of the phosphorus in dicalcium phosphate, bone meal, soft phosphatc and calcium phytate for mature wethers. J. Nutr., 70, $58-62$.

Lovg T. A., Thllmax A. D., Nelson A. B., Davis B., Wildis D., (ihllep W. D., i956. Dicalcium phosphate and soft phosphate with colloüdal clay as source of phosphorus for beef heifers. $j$. amim. Sci.. 15, III2-III8.

Long T. A., Tillman A. D., Nelson A. B., Gillue W. D., D.AVIs B., 1957. Availability of phosphorus in mineral supplements for beef cattle. J. anim. Sci., 16, $+4+-450$.

Leeker C. F., Lofgreen G. P., 106r. Effects of intake and ralcium to phosphorus ratio on absorption of these elements by sheep. J. Nutr, 74, 233-238.

Matterson L. D., Sinosen E. l'., Scott II. M., I945. Kock phosphates as phosphorus supplements for growing chicks. Poultry Sci., 24, Is8-19o.

Ic Colley E. V., Rask O. S., Becker J. F.., 1928. A study of the possible role of alummium compounds in animal plant and physiology. J. Biol. Chem., 77, 753.

MC Connell E. S., Insko W. M. (jr), Brckner G. I)., igh. Fused rock phosphate for chicks. Nulr. Abstr. Rev., 16, 236.

M.sckenzie K., I93I. The biochemistry of alumnium. II. Ficretion and abomption of aluminium in the rat. Biochem. J., 25, 287.

Menzies C. S., Richardson D., Jaker F. II., Cox R. li, ig55. Phosphoric acid as a source of phosphorus for ruminants. J. anim. Sci., 14, I 217 (abstr.).

Miller M. W., Joukovsky V. V., I953. Availability of phosphorus trom varous phosphate materiats for chicks. Poultry Sci., 32, 78-81.

Iotzok I., ARthur D., Branion H. D., 1956. Utilization of phosphorus from varnous phosphate supplements for chicks. Poultry Sci., 35, 627-649.

l'ERo R., I945. Expériences portant sur l'assimilation des phosphates tricalciques naturels finement moulus par l'organisme du porc. Ann. Agron., 15, 288-300.

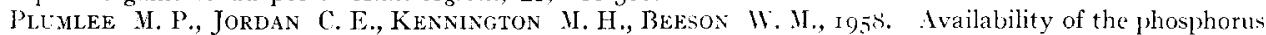
from various phosphate materials for swine. J. anim. Sci., 17, 73-88.

Raxboin L., I955. Travaux non publiés clu laboratoire de contróle biologrique des aliments de la sociéte d'hygiène alimentaire. I'aris.

Raus A., Anderson R., Cheng l., Hale W. H., Burroccils W., r95os. The validity of the artificial rumen technique in measuring phosphorus availability to cattle and sheep. $J$. anim. Sci., 15 , I 244 (Abstr.).

REINACH N., Loww J. (;., I950. The availability of the calcium and phosphorus in " lilectrotos " for bone formation. I. In the rat. Onderslepoort. J. Vet. Res., 24, 87-Iot.

Reinach N., Louw J. G., I952. The utilization of phosphorus from different sources by the rat. Onderslepoort J. Vet. Res., 25, 99-104.

RENACH N., Louw J. G., I958a. The utilization of the phosphorus trom an alummiun -.. iron rock phosphate. I. By the rat. Onderstepoort. J. Vet. Res., 27, 61 I-616.

REINACH N., Louw J. G., I 958b. The utilization of the phosphorus from an aluminium-iron rock phosphate II. By the sheep. Onderstepoort $J$. Vet. Res., 27, 61 7-624.

Richardson D., Baker F. H., Menzies C. S., Cox R. F., io5.5. Sources of phosphorus for winteringr beef heifers on dry bluestem pasture. Kans. Agric. Exp. Sta. (ir., 320.

Schreier K., Nöller H. G., I955. Stoffwechselversuche mit verschiedenen markierten Polyphosphaten. Nutr. abstr. Rev., 26, 7I 5.

Singsen E. P., ScotT H. M., I946. Poultry Sci., 25, 302.

STREET H. R., r942. Influence of aluminium sulphate and aluminium hychroxide upon the absorption of dietary phosphorus by the rat. $J$. Nutr., 24, I I I-I I 9 .

Struthers P. H., SIELING D. H., I950. Effect of organic anions on phosphate precipitation by iron and aluminium as influenced by pH. Soil Sci., 69, 205.

Thompson A., Ha.ssari) S. L., BeLl M. C., 1959. The influence of aluminium and zinc upon the absorption and retention of calcium and phosphorus in lambs. J. anim. Sci., 18, 187-197.

Tillman A. D., Brethour J. R., I958a. Ruminant utilization of sodium meta-, ortho-, and pyrophosphates, J. anim. Sci., 17, 792-796.

Tillyas A. D., BREthour J. R., I 9586 . Dicalcium phosphate and phosphoric acid as phosphorus sources for beef cattle. J. anim. Sci, 17, 100-103. 
Tillyax A. D., Brethour J. R., Ilaxsard S. L., 1959. Comparative procedures for measuring the phosphorus requirement of cattle. J. anim. Sci., 18, 249-255.

TyLER C., I948. The mineral requirements and metabolism of poultry. Calcium and phosphorus. Part. I. Nutr. abstr. Reu., 18, 261-274.

Wasserman R. I., i 960. Calcium and phosphorus interactions in nutrition and physiology. Feder. Proc., $19,636-642$.

Wentwortil R. A., Wise M. B., Simitn S. E., ig6o. Availability of the phosphorus in various sources for dairy calves (Abstr.) J. anim. Sci., 19, 1299-1 300.

Wilcox R. A., Carlson C. W., Konlmeyer W., Gastler G. F., I954. The availability of phosphorus from different sources for poults fed purified diets. Poullry Sci., 33, Io Io-Ior 4.

Wilcox R. A., Carlson C. W., Kohlmeyer W., Gastler G. F., i955. The availability of phosphorus from different sources for poults fed practical-type diets. Poultry Sci., 34, ro17-I023.

Willians D. E., MaC Leod F. I., Morrei.t E., P'ntrick H., i949. Animal feeding tests. Ind. Eng. Chem, 41, I 39 I-I 396.

Wise M. B., Smith S. E., Barnes L. L., 1958. The phosphorus requirement of calves. J. anim. Sci., $17,89-99$.

Zelter S. Z., Tisserand J. L., igoo. Valeur nutritionnelle pour l'agneauu sevré d'un phosphate aluminocalco-ferrique. Industr. Aliment. Anim., 111, 5г-59 